Pacific Journal of Mathematics

ON WEAK RESTRICTED ESTIMATES AND ENDPOINT
PROBLEMS FOR CONVOLUTIONS WITH OSCILLATING

WOLFGANG B. JURKAT AND GARY SAMPSON 


\title{
ON WEAK RESTRICTED ESTIMATES AND ENDPOINT PROBLEMS FOR CONVOLUTIONS WITH OSCILLATING KERNELS (I)
}

\author{
W. B. JURKat and G. SAMPSON
}

Throughout we consider $K(t)=e^{i|t|^{a}} /|t|^{b}, a>0, \quad a \neq 1$, $b<1$ and $t \in \boldsymbol{R}$. Here we consider for fixed $\lambda, \mu>0$ the function $B(\lambda, \mu ; K)=B(\lambda, \mu)=\sup _{\chi_{\lambda} \chi_{\mu}} \int \chi_{\lambda}(x) K * \chi_{\mu}(x) d x$ where the sup is taken over all "characteristic" functions $\chi_{\lambda}, \chi_{\mu}$ with complex signs (i.e., $\chi_{\mu}$ is a measurable function for which $\left|\chi_{\mu}\right|=1$ on $E,\left|\chi_{\mu}\right|=0$ off $E$ and $\left.|E| \leqq \mu(\mu>0)\right)$. We estimate $B(\lambda, \mu ; K)$ within constant factors from above and below. This settles the endpoint problems for these kernels, at least in the weak restricted sense.

o. Introduction. This paper is concerned with $\left(L_{p}, L_{q}\right)$-mapping properties of the operator

$$
g=K * f, \quad g(x)=\int K(x-y) f(y) d y \quad\left(x, y \in \boldsymbol{R}^{n}\right),
$$

in particular with (weak restricted) estimates

$$
\left|\int \chi_{\lambda}\left(K * \chi_{\mu}\right)\right| \leqq c_{p q} \lambda^{1 / q^{\prime}} \mu^{1 / p}\left(1 / q+1 / q^{\prime}=1\right),
$$

where, e.g., $\chi_{\mu}$ denotes a "characteristic" function with complex signs, i.e., a measurable function with $\left|\chi_{\mu}\right|=1$ on $E,\left|\chi_{\mu}\right|=0$ off $E,|E| \leqq \mu(\mu>0)$. Let us denote by $B(\lambda, \mu) \equiv B(\lambda, \mu ; K)$ the quantity

$$
\sup _{\chi_{2} \cdot \chi_{\mu}}\left|\int \chi_{\lambda}\left(K * \chi_{\mu}\right)\right|=\sup _{\chi_{2} \cdot \chi_{\mu}}\left|\iint K(x+y) \chi_{2}(x) \chi_{\mu}(y) d x d y\right|,
$$

where the sup varies over all characteristic functions $\chi_{\lambda}, \chi_{\mu}$ with fixed $\lambda>0, \mu>0$. Our present problem will be to estimate $B(\lambda, \mu)$ as closely as possible from above and below.

In earlier papers [4], [9], [13], [14] we already discussed the mapping properties for oscillating kernels. In [4] we gave, in part, the mapping properties for the kernels

$$
K(t)=\frac{e^{i|t|^{a}}}{|t|^{b}}(0 \neq t \in R) \text { with } a>0, a \neq 1, b<1
$$

except for the endpoints. By means of the function $B(\lambda, \mu)$ we settle the endpoint problems in the weak restricted sense. Furthermore, we determine $B(\lambda, \mu)$ within constant factors from above and 
below. Thus for the kernels $K$ in (2) we can determine the set $S(K)=S$ of all mapping points $(1 / p, 1 / q)$ in the Riesz triangle $0 \leqq$ $1 / q \leqq 1 / p \leqq 1$. This will imply weak as well as strong mapping properties at all boundary points except possibly at the vertices of $S$ (this is obtained through interpolation). Recently, in the paper [10; Theorem 5 and Remark 2] we were able to prove that the kernels in (2) satisfy strong mapping properties at the vertices $B$, $B^{\prime}$ and $C$ of $S$.

In [5] and along with Stein in [7], C. Fefferman solved the $\left(L^{p}, L^{q}\right)$ mapping problem for these kernels in (2) with $a<0$ (when $a<0$ we assume further that $K$ has compact support). Just recently Fefferman had pointed out to us that he also knew how to solve the mapping problem when $0<a<1$ (of course all of his results apply as well to $n$-dimensions). To be more precise the methods used by Fefferman assume that the kernels $K(t)=e^{i|t|^{a}} /(1+|t|)$ (here $t \in \boldsymbol{R}, 0<a, a \neq 1$ ) satisfy at least a regularity condition at infinity, i.e., for some $0<\theta<1$,

$$
\int_{|x| \geqq 2|y| 1 /(1-\theta)}|K(x-y)-K(x)| d x \leqq B, \quad \text { for }|y| \geqq 1,
$$

$B$ a positive constant independent of $y$. One can easily show that the kernels $K(t)=(1+|t|)^{-1} e^{i|t|^{a}}$ where $a>1$, do not satisfy such a regularity condition for any $0<\theta<1$.

Let me add that $P$. Sjölin in [17] has solved some of these mapping problems in $n$-dimensions.

1. An interpolation theorem with respect to the kernel. The usual interpolation theorems refer to two function spaces and a corresponding decomposition $f=f_{1}+f_{2}$. Here we consider a decomposition of the kernel:

$$
K=K_{1}+K_{2} \text {. }
$$

We make use of the decreasing rearrangement $K^{*}$ of $K$ (if it exists), so that

$$
\sup _{\chi_{\mu}}\left|\int K(x) \chi_{\mu}(x) d x\right|=\int_{0}^{\mu} K^{*}(t) d t \quad\left(x \in \boldsymbol{R}^{n}, t \in \boldsymbol{R}\right) .
$$

We also make use of the (distributional) Fourier transform

$$
\hat{K}(x)=\int e^{2 i x \cdot y} K(y) d y .
$$

With these notations we have the following

THEOREM 1. If $K_{1}^{*}$ exists and $\hat{K}_{2}$ is bounded then 


$$
B(\lambda, \mu) \leqq \lambda \int_{0}^{\mu} K_{1}^{*}(t) d t+(\lambda \mu)^{1 / 2}\left\|\hat{K}_{2}\right\|_{\infty}
$$

Since $B(\lambda, \mu)=B(\mu, \lambda)$ there is a second inequality with $\lambda$ and $\mu$ interchanged.

Proof. Corresponding to the decomposition of $K$ we have

$$
B(\lambda, \mu ; K) \leqq B\left(\lambda, \mu ; K_{1}\right)+B\left(\lambda, \mu ; K_{2}\right) .
$$

Here the first term can be estimated using

$$
\left\|K_{1} * \chi_{\mu}\right\|_{\infty} \leqq \int_{0}^{\mu} K_{1}^{*}(t) d t
$$

and the second term can be estimated using

$$
\left\|K_{2} * \chi_{\mu}\right\|_{2} \leqq\left\|\hat{K}_{2}\right\|_{\infty} \mu^{1 / 2}
$$

REMARK. Observe that

$$
K_{1}^{* *}(\mu)=\frac{1}{\mu} \int_{0}^{\mu} K_{1}^{*}(t) d t \leqq \frac{1}{\lambda} \int_{0}^{\lambda} K_{1}^{*}(t) d t \quad \text { for } \quad \lambda \leqq \mu,
$$

so that interchanging $\lambda$ with $\mu$ produces a weaker inequality in the case $\lambda \leqq \mu$. Example (2) can be used to show that the "right" decomposition of $K$ gives sharp estimates for all $\lambda$, $\mu$ which is rather surprising in view of the two simple estimates used. Also note that

$$
\|g\|_{q}^{*}=\sup _{\lambda>0, \chi_{\lambda}} \lambda^{-1 / q^{\prime}}\left|\int \chi_{\lambda} g\right|, \quad 1<q<\infty
$$

defines a weak $q$-norm of $g$ (which is equivalent to the usual weak $q$-norm). Therefore

$$
N_{q}(\mu)=\sup _{\chi_{\mu}}\left\|K * \chi_{\mu}\right\|_{q}^{*}=\sup _{\lambda>0} \lambda^{-1 / q^{\prime}} B(\lambda, \mu)
$$

can be calculated via $B(\lambda, \mu)$. Note that $N_{q}(\mu)$ is a logarithmically convex function in $1 / q$ ( $\mu$ fixed) since it is a sup of such functions.

2. Upper estimates for $B$. From now on we consider the kernel

$$
K(t)=\frac{e^{i|t|^{a}}}{|t|^{b}}(0 \neq t \in R) \quad \text { with } \quad a>0, a \neq 1, b<1 .
$$

By $c, c_{1}, c_{2}, \cdots$ we generically denote suitable positive constants which depend only on $a$ and $b$. We introduce $K_{w}(w>0), K^{w}(w \geqq 0)$, and $K_{u, v}(0 \leqq u<v \leqq \infty)$ by $K_{w}^{(t)}=K(t)$ for $|t|<w, K^{w}=K$ for 
$|t| \geqq w, K_{u, v}=K$ for $u \leqq|t|<v$ and $K_{w}=0, K^{w}=0, K_{u, v}=0$ elsewhere.

By standard estimates (van der Corput, eg.,) we obtain for all real $x$ and all (admissible) $w$

$$
\begin{array}{ll}
\left|\hat{K}^{w}(x)\right| \leqq c(w+1)^{1-(a / 2)-b} & \text { if } \quad b \geqq 1-\frac{a}{2}, \\
\left|\hat{K}_{w}(x)\right| \leqq c w^{1-\{\alpha / 2)-b} & \text { if } \quad b<1-\frac{a}{2} .
\end{array}
$$

Furthermore

$$
\begin{aligned}
& \int_{0}^{\mu}\left(K_{w}\right)^{*} \leqq \begin{cases}c \min \left(\mu^{1-b}, w^{1-b}\right) & \text { if } \quad b \geqq 0 \\
c \min \left(\mu w^{-b}, w^{1-b}\right) & \text { if } \quad b<0,\end{cases} \\
& \int_{0}^{\mu}\left(K^{w}\right)^{*} \leqq c \min \left(\mu^{1-b}, \mu w^{-b}\right) \text { if } \quad b \geqq 0 .
\end{aligned}
$$

Accordingly we distinguish between the four cases

$$
\begin{array}{ll}
b \geqq 1-\frac{a}{2}, & b \geqq 0, \\
b \geqq 1-\frac{a}{2}, & b<0 \text { (implies } a>2 \text { ), } \\
b<1-\frac{a}{2}, & b \geqq 0 \text { (implies } a<2 \text { ), } \\
b<1-\frac{a}{2}, & b<0 .
\end{array}
$$

In case (IV) there will be no nontrivial estimates. In the other cases we employ Theorem 1. Each of the cases (I), (II), (III) will be subdivided according to $\lambda \leqq \mu^{1-a}$ (subscript 1 ) or $\lambda \geqq \mu^{1-a}$ (subscript 2). Our estimates for $B(\lambda, \mu)=B(\lambda, \mu ; a, b)$ are as follows:

Case I. Using $K_{1}=K, K_{2}=0$, we obtain

$$
B(\lambda, \mu) \leqq c \lambda \mu^{1-b}, \quad\left[B(\lambda, \mu) \leqq c \mu \lambda^{1-b}\right] .
$$

The first inequality is better than the second one if $\lambda \leqq \mu$; they agree if $b=0$.

Using $K_{1}=K_{w}, K_{2}=K^{w}$ with $w=(\mu / \lambda)^{1 / a} \leqq \mu$, we obtain

$$
\begin{aligned}
B(\lambda, \mu) & \leqq c \lambda w^{1-b}+c(\lambda \mu)^{1 / 2} w^{1-(a / 2)-b}, \\
& \leqq c \lambda^{(a+b-1) / a} \mu^{(1-b) / a} \text { in case }\left(\mathrm{I}_{2}\right) .
\end{aligned}
$$

Since 


$$
\lambda \mu^{1-b} \leqq \lambda^{(a+b-1) / a} \mu^{(1-b) / a} \quad \text { iff } \quad \lambda \leqq \mu^{1-a}
$$

the last inequality above holds also in case $\left(I_{1}\right)$ since we have the better inequality (i) there. So we have without restrictions [by symmetry]

$$
B(\lambda, \mu) \leqq c \lambda^{(a+b-1) / a} \mu^{(1-b) / a}, \quad\left[B(\lambda, \mu) \leqq c \mu^{(a+b-1) / a} \lambda^{(1-b) / a}\right] .
$$

Here the left inequality is better for $\lambda \leqq \mu$ since $a+b-1 / a \geqq$ $1-b / a$; the inequalities agree if $b=1-(a / 2)$.

In total we have four inequalities in case (I). They come in pairs, and we may always pick the first one, since $\lambda \leqq \mu$ can be assumed by symmetry. Then (i) is relevant (better) for $\left(\mathrm{I}_{1}\right)$ and (ii) for $\left(I_{2}\right)$.

Case (II). Letting $K_{1}=K_{w}, K_{2}=K^{w}$ with $w=(\mu / \lambda)^{1 / a} \leqq \mu$, we obtain

$$
\begin{aligned}
B(\lambda, \mu) & \leqq c \lambda w^{1-b}+c(\lambda \mu)^{1 / 2} w^{1-(a / 2)-b} \\
& \leqq c \lambda^{(a+b-1) / a} \mu^{(1-b) / a} \text { in case }\left(\mathrm{II}_{2}\right) .
\end{aligned}
$$

With $w \geqq \mu$ we obtain

$$
\begin{aligned}
& B(\lambda, \mu) \leqq c \lambda \mu w^{-b}+c(\lambda \mu)^{1 / 2} w^{1-(a / 2)-b}, \quad w=(\lambda \mu)^{-1 /(a-2)} \geqq \mu, \\
& B(\lambda, \mu) \leqq c(\lambda \mu)^{(a+b-2) /(a-2)} \quad \text { in case }\left(\mathrm{II}_{1}\right) .
\end{aligned}
$$

Observe that

$$
(\lambda \mu)^{(a+b-2) /(a-2)} \leqq \lambda^{(a+b-1) / a} \mu^{(1-b) / a} \quad \text { iff } \quad \lambda \leqq \mu^{1-a}
$$

so that the first inequality extends to $\left(\mathrm{II}_{1}\right)$ and the second inequality to $\left(\mathrm{II}_{2}\right)$. Thus we get [by symmetry] the three unrestricted inequalities

$$
\begin{gathered}
B(\lambda, \mu) \leqq c \lambda^{(a+b-1) / a} \mu^{(1-b) / a}, \quad\left[B(\lambda, \mu) \leqq c \mu^{(a+b-1) / a} \lambda^{(1-b) / a}\right], \\
B(\lambda, \mu) \leqq c(\lambda \mu)^{(a+b-2) /(a-2)} .
\end{gathered}
$$

Of the first two the first one is better for $\lambda \leqq \mu$ which can always be assumed. Then (ii) is relevant for $\left(\mathrm{II}_{2}\right)$ and (iii) for $\left(\mathrm{II}_{1}\right)$.

Case (III). Letting $K_{1}=K, K_{2}=0$, we obtain

$$
B(\lambda, \mu) \leqq c \lambda \mu^{1-b}, \quad\left[B(\lambda, \mu) \leqq c \mu \lambda^{1-b}\right] .
$$

The first inequality is better than the second one if $\lambda \leqq \mu$.

Letting $K_{1}=K^{w}, K_{2}=K_{w}$ with $w=(\lambda \mu)^{1 /(2-a)} \geqq \mu$ we obtain

$$
\begin{aligned}
B(\lambda, \mu) & \leqq c \lambda \mu w^{-b}+c(\lambda \mu)^{1 / 2} w^{1-(a / 2)-b}, \\
& \leqq c(\lambda \mu)^{(2-a-b) /(2-a)} \quad \text { in case }\left(\mathrm{II}_{2}\right) .
\end{aligned}
$$


Since

$$
\lambda \mu^{1-b} \leqq(\lambda \mu)^{(2-a-b) /(2-a)} \text { if } \lambda \leqq \mu^{1-a},
$$

the last inequality above extends to $\left(\mathrm{III}_{1}\right)$, i.e., we have without restrictions

$$
B(\lambda, \mu) \leqq c(\lambda \mu)^{(2-a-b) /(2-a)} .
$$

Again, (i) is relevant for (III $)_{1}$ and (iii) for $\left(\mathrm{III}_{2}\right)$.

Thus we see that if $\lambda \leqq \mu$ then in each of the cases (I), (II), (III) there is just one of the inequalities (i), (ii), (iii) relevant for $\lambda \leqq \mu^{1-a}$ and one for $\lambda \geqq \mu^{1-a}$. This suggests defining the following explicit function $\widetilde{B}(\lambda, \mu ; a, b)$ for $\lambda \leqq \mu$ :

$$
\begin{aligned}
& \widetilde{B}=\lambda \mu^{1-b} \text { for }\left(\mathrm{I}_{1}\right), \quad \widetilde{B}=\lambda^{(a+b-1) / a} \mu^{(1-b) / a} \text { for }\left(\mathrm{I}_{2}\right) ; \\
& \widetilde{B}=(\lambda \mu)^{(a+b-2) /(a-2)} \quad \text { for }\left(\mathrm{II}_{1}\right), \quad \widetilde{B}=\lambda^{(a+b-1) / a} \mu^{(1-b) / a} \text { for }\left(\mathrm{II}_{2}\right) ; \\
& \widetilde{B}=\lambda \mu^{1-b} \text { for }\left(\mathrm{III}_{1}\right), \quad \widetilde{B}=(\lambda \mu)^{(2-a-b) /(2-a)} \text { for }\left(\mathrm{III}_{2}\right) ;
\end{aligned}
$$

along the dividing line $\lambda=\mu^{1-a}$ both definitions in (I), (II), (III) give the same value. For $\lambda \geqq \mu$ we define $\widetilde{B}(\lambda, \mu)$ by symmetry. Finally, we set $\widetilde{B}=\infty$ in case (IV). Then we can summarize the upper estimates as

TheOREM 2. Always $B(\lambda, \mu) \leqq c \widetilde{B}(\lambda, \mu)$.

REMARK. We note that $K$ can be replaced by $K_{u, v}$ in all upper estimates, and that these hold true uniformly in $u$ and $v$.

There is a more compact, but less explicit way to define $\widetilde{B}(\lambda, \mu)$ : In case (I) we had the four general upper estimates

$$
\lambda \mu \mu^{1-b}, \quad \mu \lambda^{1-b}, \quad \lambda^{(a+b-1) / a} \mu^{(1-b) / a}, \quad \mu \mu^{(a+b-1) / a} \lambda^{(1-b) / a},
$$

except for the constant $c$. Now $\widetilde{B}$ is simply the minimum of these four functions and similarly in the cases (II), (III).

3. Lower estimates for $B$. Here we prove the opposite inequality for $B(\lambda, \mu ; a, b)$.

Theorem 3. Always $B(\lambda, \mu) \geqq c \widetilde{B}(\lambda, \mu)$.

Thus the order of magnitude of $B(\lambda, \mu)$ is determined for all $\lambda, \mu$. Note that the special case $a=2, b=0$ is essentially the case of the Fourier transform. The proof of Theorem 3 is based on the following result, where 


$$
\psi_{\mu}(x)=\int_{-\infty}^{\infty} K(x+t) \chi_{\mu}(t) d t \quad(x, t \in R)
$$

and $\chi_{\mu}$ is our characteristic function.

Proposition. There are constants $c_{1}$, $c_{2}$ such that to each pair of parameters $\delta, T$ with $0<\delta \leqq \mu, T \geqq \delta$ there exists a characteristic function $\chi_{\mu}$ satisfying

$$
\left|\left\{x:\left|\psi_{\mu}(x)\right|>c_{1} \delta T^{-b}\right\}\right| \geqq c_{2} \min \left(T, \delta^{-1} T^{2-a}\right) .
$$

In case that $a+b>1$ this can be improved to

$$
\left|\left\{x:\left|\psi_{\mu}(x)\right|>c_{1} \delta T^{-b}\right\}\right| \geqq c_{2} \mu \delta^{-1} \min \left(T, \delta^{-1} T^{2-a}\right) .
$$

Proof. Let

$$
\chi_{\delta}(t)= \begin{cases}e^{-i t^{a}} & \text { for } t \in(T, T+\delta) \\ 0 & \text { elsewhere }\end{cases}
$$

and observe that for $0<x \leqq T$

$$
\left|\psi_{\delta}(x)\right|=\left|\int_{T}^{T+\delta} K(x+t) \chi_{\delta}(t) d t\right|=\int_{T}^{T+\delta} \frac{e^{i \Delta(x, t)}}{(x+t)^{b}} d t \mid,
$$

where the second difference

$$
\Delta(x, t)=\left[(x+t)^{a}-t^{a}\right]-\left[(x+T)^{a}-T^{a}\right]=\int_{0}^{x} d \xi \int_{T}^{t} d \tau a(a-1)(\xi+\tau)^{a-2}
$$

can be estimated as

$$
|\Delta| \leqq c x \delta T^{a-2} \leqq 1 \quad \text { if } \quad x \leqq \frac{1}{c} \delta^{-1} T^{2-a} .
$$

Hence we obtain

$$
\left|\psi_{\delta}(x)\right|>c_{1} \delta T^{-b} \text { for } 0<x<X=c_{2} \min \left(T, \delta^{-1} T^{2-a}\right) .
$$

Taking $\chi_{\mu}=\chi_{\delta}$ the first part of the proposition is clear.

Now assume $a+b>1$ and observe that

$$
\psi_{\delta}(x)= \pm \int_{T}^{T+\delta} \frac{e^{-i t^{a}}}{i a|x+t|^{a+b-1}} d\left(e^{i|x+t|^{a}}\right) \longrightarrow 0
$$

as $|x| \rightarrow+\infty$ using partial integration. We set

$$
\chi_{\mu}(t)=\sum_{j=1}^{k} \chi_{\delta}\left(t+x_{j}\right), \quad \psi_{\mu}(x)=\sum_{j=1}^{k} \psi_{\delta}\left(x-x_{j}\right),
$$

where the integer $k$ is defined by 


$$
k=\left[\frac{\mu}{\delta}\right] \geqq 1,
$$

the real numbers $x_{j}$ are selected so that

$$
\left|x_{i}-x_{j}\right| \geqq \max (\delta, X+d) \text { for } i \neq j,
$$

and $d>0$ is selected so that

$$
\left|\psi_{\delta}(x)\right| \leqq \frac{c_{1}}{2 k} \delta T^{-b} \quad \text { for } \quad|x| \geqq d .
$$

Observe that the supports of $\chi_{\hat{o}}\left(t+x_{j}\right)$ are disjoint and that the support of the characteristic function $\chi_{\mu}$ has measure $k \delta \leqq \mu$; furthermore

$$
\psi_{\mu}(x)=\int K(x+t) \chi_{\mu}(t) d t
$$

Finally, if $x-x_{i}$ is in the interval $I=(0, X)$ then $\left|x-x_{j}\right| \geqq d$ for $j \neq i$, hence

$$
\left|\psi_{\mu}(x)\right|>c_{1} \delta T^{-b}-(k-1) \frac{c_{1}}{2 k} \delta T^{-b} \geqq \frac{c_{1}}{2} \delta T^{-b} \quad \text { for } \quad x \in x_{i}+I .
$$

Since the intervals $x_{i}+I$ are disjoint we have

$$
\left|\left\{x:\left|\psi_{\mu}(x)\right|>\frac{c_{1}}{2} \delta T^{-b}\right\}\right| \geqq k X,
$$

which is the second claim with changed (smaller) constants.

Integrating the function $\left|\psi_{\mu}\right|$ over a suitable subset of size $\lambda$ gives the following

Corollary. Assume $0<\delta \leqq \mu, T \geqq \delta$. Then

$$
B(\lambda, \mu) \geqq c_{1} \lambda \delta T^{-b}
$$

if $\lambda \leqq c_{2} \min \left(T, \delta^{-1} T^{2-a}\right)$; in case that $a+b>1$ the estimate (3) holds even in the larger range $\lambda \leqq c_{2} \mu \delta^{-1} \min \left(T, \delta^{-1} T^{2-a}\right)$.

REMARK. Note that in the first case of the corollary the supports of $\chi_{\mu}$ and $\chi_{\lambda}$ can be taken to be single intervals, namely $(T, T+\delta)$ and $(0, X)$ respectively. In the second case we take $k$ translates of each of these intervals which are spread apart by a minimum amount (at least the size of these intervals).

In order to apply the corollary we fix $\lambda, \mu$ arbitrarily and select optimal values of $\delta, T$ among those which are permitted. In terms of $\log \delta$ and $\log T$ this is a simple linear programming problem. 
In case (IV), cf. $§ 2$, we get

$$
B(\lambda, \mu)=\infty \text { for all } \lambda, \mu,
$$

since (IV) can be broken down into the subcases

$\left(\mathrm{IV}_{1}\right) \quad a \leqq 2, \quad b<0\left[\delta=\min \left(\mu, c_{2} \lambda^{-1}\right), T \longrightarrow+\infty\right]$,

$\left(\mathrm{IV}_{2}\right) \quad a>2, \quad b<2-a\left[\delta=c_{2} \lambda^{-1} T^{2-a}, T \longrightarrow+\infty\right]$,

$\left(\mathrm{IV}_{3}\right)$

$$
a>2, \quad b<1-\frac{a}{2},
$$

$$
b \geqq 2-a(>1-a)\left[\delta=\left(\frac{c_{2} \mu}{\lambda}\right)^{1 / 2} T^{1-(a / 2)}, T \longrightarrow+\infty\right] .
$$

In what follows we assume $\lambda \leqq \mu$ since $B(\lambda, \mu)$ is symmetric. Remember that (i) was relevant for $\left(\mathrm{I}_{1}\right)$ and $\left(\mathrm{III}_{1}\right)$, i.e., if $b \geqq 0$ and $\lambda \leqq \mu^{1-a}$. We will now show that

$$
B(\lambda, \mu) \geqq c \lambda \mu^{1-b} \quad \text { if } \quad \lambda \leqq \mu^{1-a},
$$

so that (i) is optimal where it is relevant: simply choose in the corollary

$$
T=\frac{\mu}{c_{2}}, \quad \delta=\min \left(1, c_{2}^{-1}, c_{2}^{a-1}\right) \mu .
$$

Remember that (ii) was relevant for $\left(\mathrm{I}_{2}\right)$ and $\left(\mathrm{II}_{2}\right)$, i.e., if $b \geqq$ $1-(a / 2)$ and $\lambda \geqq \mu^{1-a}$. We will now show that

$$
B(\lambda, \mu) \geqq c \lambda^{(a+b-1) / a} \mu^{(1-b) / a} \quad \text { if } \quad b \geqq 1-\frac{a}{2}, \quad \lambda \geqq \mu^{1-a},
$$

so that (ii) is optimal where it is relevant: since $a+b>1$, $(\mu / \lambda)^{1 / a} \leqq \mu$ simply choose in the corollary

$$
T=\left(\frac{\mu}{\lambda}\right)^{1 / a}, \quad \delta=\min \left(1, c_{2}, c_{2}^{1 / 2}\right)\left(\frac{\mu}{\lambda}\right)^{1 / a} .
$$

Finally, remember that (iii) was relevant in $\left(\mathrm{II}_{1}\right)$ and $\left(\mathrm{III}_{2}\right)$, i.e., in case (II) with $\lambda \leqq \mu^{1-a}$ and in case (III) with $\lambda \geqq \mu^{1-a}$. We will now show that

$$
B(\lambda, \mu) \geqq c(\lambda \mu)^{(a+b-2) /(a-2)} \quad \text { if } \quad\left\{\begin{array}{l}
a>2, \lambda \leqq \mu^{1-a} \\
\text { or } a<2, \lambda \geqq \mu^{1-a},
\end{array}\right.
$$

so that (iii) is optimal where it is relevant: since $(\lambda \mu)^{1 /(2-a)} \geqq \mu$ simply choose in the corollary

$$
T=\frac{1}{c_{2}}(\lambda \mu)^{1 /(2-a)}, \quad \delta=\min \left(1, c_{2}^{-1}, c_{2}^{a-1}\right) \mu .
$$


The lower estimates are summarized in Theorem 3.

4. Applications. In this section we will determine the weak restricted mapping set $S(K)$ as consequence of our estimates for $B(\lambda, \mu ; a, b)$, and we will also determine the order of magnitude of $N_{q}(\mu)$.

We begin with $S(K)$. It is convenient to introduce the following four linear functions in $1 / q$ :

$$
\begin{aligned}
& \gamma_{1}=1-b+\frac{1}{q}, \quad \gamma_{2}=1-b+(1-a) / q, \\
& \gamma_{3}=\frac{1}{q}, \quad \gamma_{4}=\frac{1-a-b+1 / q}{1-a} .
\end{aligned}
$$

A point $(1 / p, 1 / q)$ with $1 \leqq p \leqq \infty, 1<q<\infty$ is of weak restricted type if (1) holds, i.e., if both

$$
B(\lambda, \mu) \leqq c_{p q} \lambda^{1 / q^{\prime}} \mu^{1 / p}, \quad B(\lambda, \mu) \leqq c_{p q} \mu^{1 / q^{\prime}} \lambda^{1 / p}
$$

hold (by symmetry). Using the simple cases $\lambda=\mu^{1-a}$ and $\lambda=\mu$ of Theorem 3 we obtain the following necessary conditions

$$
\begin{aligned}
& \mu^{1-a} \mu^{1-b} \leqq c_{p q} \mu^{(1-a) / q^{\prime}} \mu^{1 / p} \quad \text { for } \quad \mu \geqq 1, \\
& \mu^{1-a} \mu^{1-b} \leqq c_{p q} \mu^{1 / q^{\prime}} \mu^{(1-a) / p} \quad \text { for } \quad \mu \geqq 1, \\
& \mu \mu^{1-b} \leqq c_{p q} \mu^{1 / q^{\prime}} \mu^{1 / p} \quad \text { for } \quad \mu \leqq 1(b \geqq 0) .
\end{aligned}
$$

By letting $\mu \rightarrow+\infty$ resp. $\mu \rightarrow+0$ we get the three inequalities

$$
\begin{aligned}
& \gamma_{2} \leqq \frac{1}{p}, \quad \gamma_{4} \leqq \frac{1}{p}(\text { if } a<1) \quad \text { or } \quad \gamma_{4} \geqq \frac{1}{p}(\text { if } a>1), \\
& \gamma_{1} \geqq \frac{1}{p}(\text { if } b \geqq 0) .
\end{aligned}
$$

We can add to this the well known necessary condition $q \geqq p$, i.e., $\gamma_{3} \leqq 1 / p$. We are going to show that these inequalities are not only necessary but also sufficient (for $1<q<\infty$ ), i.e., they describe the set $S(K)$.

To get a clearer geometric picture of the situation we introduce the following five points in the $(1 / p, 1 / q)$-plane:

$$
\begin{aligned}
& A=(1-b, 0), \quad A^{\prime}=(1, b) ; \\
& B=\left(\frac{1-b}{a}, \frac{1-b}{a}\right), \quad B^{\prime}=\left(\frac{a+b-1}{a}, \frac{a+b-1}{a}\right)
\end{aligned}
$$

and

$$
C=C^{\prime}=\left(\frac{a+b-2}{a-2}, \frac{b}{2-a}\right) \text { if } a \neq 2 .
$$


Furthermore we consider the straight lines

$$
\begin{aligned}
& l_{1}: \gamma_{1}=\frac{1}{p} \text { passing through } A, A^{\prime}, \\
& l_{2}: \gamma_{2}=\frac{1}{p} \text { passing through } A, B, C, \\
& l_{3}: \gamma_{3}=\frac{1}{p} \text { passing through } B, B^{\prime}, \\
& l_{4}: \gamma_{4}=\frac{1}{p} \text { passing through } A^{\prime}, B^{\prime}, C^{\prime}=C .
\end{aligned}
$$

Next we describe the set characterized by the linear inequalities above:

In case (I) we have the closed quadrilateral $\left[A, A^{\prime}, B^{\prime}, B\right]$ except the vertex $A$ where $q=\infty$ (see Figure 1). Outside the quadrilateral there are no mapping points as we have seen. The vertices $A, A^{\prime}$ correspond directly to the inequalities (i); thus $A$ is of strong restricted type and $A^{\prime}$ is of weak type. Similarly the vertices $B, B^{\prime}$ correspond directly to the inequalities (ii). Then all the points of the quadrilateral are of weak restricted type by trivial convexity.

In case (II) we have the closed triangle $\left[B, C, B^{\prime}\right]$, see Figure 2. Again the vertices correspond to our main upper estimates, in particular $C$ corresponds to (iii).

In case (III) we have the closed triangle $\left[A, A^{\prime}, C\right]$ except $A$, see Figure 3.

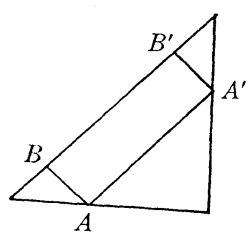

Figure 1 $(a=3, b=1 / 2)$

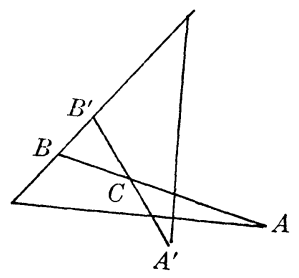

Figure 2 $(a=4, b=-1 / 2)$

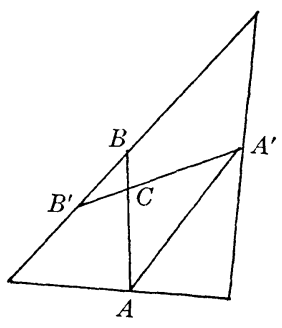

Figure 3 $(a=2 / 3, b=1 / 2)$

In case (IV) the set $S(K)$ is empty. The set $S(K)$ can degenerate into a line segment or a single point. We summarize these results as follows:

THEOREM 4. The weak restricted mapping set $S(K)$ is in case (I) the closed quadrilateral $\left[A, A^{\prime}, B^{\prime}, B\right]$ except $A$, in case (II) the closed triangle $\left[B, C, B^{\prime}\right]$ in case (III) the closed triangle $\left[A, A^{\prime}, C\right]$ 
except $A$, and empty in case (IV).

REMARKs. Observe that the boundary of $S(K)$ consists of line segments which are neither horizontal nor vertical. Since the endpoints are of weak restricted type or strong restricted type the interior points of these line segments must be strong mapping points by the convexity theorem of Stein-Weiss. The same is true for the interior of $S(K)$ anyway. So the only points which need further clarification with regard to their mapping character are the vertices $A, A^{\prime}, B, B^{\prime}, C$. It is easy to see that $A$ is strong restricted but not strong and that $A^{\prime}$ is weak but not strong $(b>0)$. Hence only $B, B^{\prime}, C$ present a problem. We will show in a subsequent paper that $C$ is strong also, which leaves only the character of $B, B^{\prime}$ partially undecided. This is settled in [10].

Finally we will determine the precise order of magnitude of $N_{q}(\mu)$. This gives a more detailed picture of the mapping properties and is based on the calculation of

$$
\tilde{N}_{q}(\mu)=\sup _{\lambda>0} \lambda^{-1 / q^{\prime}} \widetilde{B}(\lambda, \mu) .
$$

Since $\widetilde{B}$ is explicit one can work out $\widetilde{N}_{q}$ explicitly also. The calculation is lengthy, but entirely elementary; so we will drop the details. We find that whenever $\widetilde{N}_{q}<\infty$ then it is of the form

$$
\tilde{N}_{q}(\mu)=\mu^{\alpha_{q}}(\mu \leqq 1), \quad \tilde{N}_{q}(\mu)=\mu^{\beta_{q}}(\mu \geqq 1) .
$$

To describe $\widetilde{N}_{q}$ we distinguish between $a<1$ and $a>1$ in our cases (I), (II), (III).

In case (I) with $a<1$ :

If $1 / q>b$ then $\widetilde{N}_{q}(\mu)=\infty$ for all $\mu$; if $1 / q \leqq b$ then $\widetilde{N}_{q}<\infty$ and $\alpha_{q}=\gamma_{1}, \beta_{q}=\max \left(\gamma_{2}, \gamma_{3}, \gamma_{4}\right)$.

In case (I) with $a>1$ :

If $1 / q>(a+b-1) / a$ then $\widetilde{N}_{q}(\mu)=\infty$ for all $\mu$; if $1 / q \leqq(a+b-1) / a$ then $\tilde{N}_{q}<\infty$ and $\alpha_{q}=\min \left(\gamma_{1}, \gamma_{4}\right), \beta_{q}=\max \left(\gamma_{2}, \gamma_{3}\right)$.

In case (II), where automatically $a>2$ :

If $1 / q<-b /(a-2)$ or $1 / q>(a+b-1) / a$ then $\tilde{N}_{q}(\mu)=\infty$ for all $\mu$; if $-b /(a-2) \leqq 1 / q \leqq(a+b-1) / a$ then $\tilde{N}_{q}<\infty, \quad \alpha_{q}=\gamma_{4}, \quad \beta_{q}=$ $\max \left(\gamma_{2}, \gamma_{3}\right)$.

In case (III) with $a<1$ :

If $1 / q>b$ then $\tilde{N}_{q}(\mu)=\infty$ for all $\mu$; if $1 / q \leqq b$ then $\widetilde{N}_{q}<\infty$ and $\alpha_{q}=\gamma_{1}, \beta_{q}=\max \left(\gamma_{2}, \gamma_{4}\right)$.

In case (III) with $a>1$ :

If $1 / q>b /(2-a)$ then $\widetilde{N}_{q}(\mu)=\infty$ for all $\mu$; if $1 / q \leqq b /(2-a)$ then $\widetilde{N}_{q}<\infty$ and $\alpha_{q}=\min \left(\gamma_{1}, \gamma_{4}\right), \beta_{q}=\gamma_{2}$.

In case (IV): $\tilde{N}_{q}(\mu)=\infty$ for all $\mu$. 
Note that also $\tilde{N}_{q}(\mu)$ is logarithmically convex in $1 / q$. In view of Theorems 2 and 3 we have

THEOREM 5. Always $c_{3} \tilde{N}_{q}(\mu) \leqq N_{q}(\mu) \leqq c_{4} \tilde{N}_{q}(\mu)$.

This determination of $N_{q}$ will, of course, give the mapping set $S(K)$ again. But it is interesting to see that conversely the linear forms $\gamma_{1}, \gamma_{2}, \gamma_{3}, \gamma_{4}$ which correspond to the line segments of the boundary of $S(K)$ turn up in the exponents $\alpha_{q}$ and $\beta_{q}$ of $\widetilde{N}_{q}$.

\section{REFERENCES}

1. Carleson and P. Sjölin, Oscillatory integrals and a multiplier problem for the disc, Studia Math., 44 (1972), 287-299.

2. R. R. Coifman and G. Weiss, Extensions of Hardy spaces and their use in analysis, Bull. of the Amer. Math. Soc., 83, No. 4, (July 1977).

3. M. G. Cowling and J. J. F. Fournier, Inclusions and noninclusions of spaces of convolution operators, Trans. of the Amer. Math. Soc., 221 (1976), 59-95.

4. V. Drobot, A. Naparstek and G. Sampson, $\left(L_{p}, L_{q}\right)$ mapping properties of convolution transforms, Studia, Math., 55 (1976), 41-70.

5. C. Fefferman, Inequalities for strongly singular convolution operators, Acta Math., 124 (1970), 9-36.

6. — A note on spherical summation multipliers, Israel J. Math., 15 (1973), 44-52.

7. C. Fefferman and E. M. Stein, $H^{p}$ spaces of several variables, Acta Math., 129 (1972), 137-193.

8. L. Hörmander, Oscillatory integrals and multipliers in $F L^{p}$, Acta Math., 11 (1973), $1-11$.

9. W. B. Jurkat and G. Sampson, The $L^{p}$ mapping problem for well-behaved convolutions, Studia Math., 65 (1979), 227-238.

10. The complete solution to the $\left(L^{p}, L^{q}\right)$ mapping problem for a class of oscillating kernels, Indiana U. Math. J., 30 (1981), 403-413.

11. R. O'Neil, Adjunct operators and interpolation of linear operators, abstract spaces and approximation, Proceedings of the Oberwohlfach Conference, July (1968), 99-106.

12. J. C. Peral and A. Torchinsky, Multipliers in $H^{p}\left(R^{n}\right), 0<p<\infty$, Ark. Mat., 17 (1979), 225-235.

13. G. Sampson, A note on weak estimates for oscillating kernels, Studia Math., (to appear).

14. More on weak estimates for oscillating kernels, Indiana Univ. J., 28 (1979), 501-505.

15. P. Sjölin, Convergence a.e. of certain singular integrals and multiple Fourier series, Ark. Mat., 9 (1971), 65-90.

16. $\longrightarrow, L^{p}$ estimates for strongly singular convolution operators in $R^{n}$, Ark. Mat., 14 (1976), 59-94.

17. - Convolution with oscillating kernels, Indiana U. Math. J., 30 (1981), 47-56.

18. E. M. Stein and G. Weiss, An extension of a theorem of Marcinkiewicz and some of its applications, J. Math. Mech., 8 (1959), 263-284.

19. M. Zafran, Multiplier transformations of weak type, Ann. of Math., (2), 101 (1975), 34-44.

20. A. Zygmund, Trigonometric Series, 2nd ed., Vols. 1 and 2, Cambridge Univ. Press, 1959 . 
Received February 16, 1979 and in revised form December 15, 1980. The research of the first author was supported in part by the National Science Foundation.

SYRACUSE UNIVERSITY

SyRACUSE, NY 13210 


\section{PACIFIC JOURNAL OF MATHEMATICS}

\section{EDITORS}

DoNALD BABBITT (Managing Editor)

University of California

Los Angeles, CA 90024

Hugo Rossi

University of Utah

Salt Lake City, UT 84112

C. C. MOORE and ANDREw OGG

University of California

Berkeley, CA 94720
J. DugundJI

Department of Mathematics

University of Southern California

Los Angeles, CA 90007

R. FinN and J. Milgram

Stanford University

Stanford, CA 94305

ASSOCIATE EDITORS
R. ARENS
E. F. BECKENBACH
B. H. NeumanN
F. WOLF
K. YoshidA

\section{SUPPORTING INSTITUTIONS}

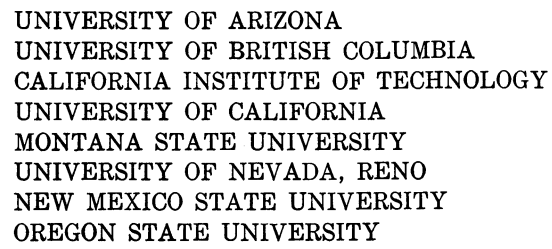

UNIVERSITY OF ARIZONA

UNIVERSITY OF BRITISH COLUMBIA

CALIFORNIA INSTITUTE OF TECHNOLOGY

UNIVERSITY OF CALIFORNIA

MONTANA STATE UNIVERSITY

UNIVERSITY OF NEVADA, RENO

NEW MEXICO STATE UNIVERSITY OREGON STATE UNIVERSITY

\author{
UNIVERSITY OF OREGON \\ UNIVERSITY OF SOUTHERN CALIFORNIA \\ STANFORD UNIVERSITY \\ UNIVERSITY OF HAWAII \\ UNIVERSITY OF TOKYO \\ UNIVERSITY OF UTAH \\ WASHINGTON STATE UNIVERSITY \\ UNIVERSITY OF WASHINGTON
}

The Supporting Institutions listed above contribute to the cost of publication of this Journal, but they are not owners or publishers and have no responsibility for its content or policies.

Mathematical papers intended for publication in the Pacific Journal of Mathematics should be in typed form or offset-reproduced, (not dittoed), double spaced with large margins. Please do not use built up fractions in the text of the manuscript. However, you may use them in the displayed equations. Underline Greek letters in red, German in green, and script in blue. The first paragraph or two must be capable of being used separately as a synopsis of the entire paper. Please propose a heading for the odd numbered pages of less than 35 characters. Manuscripts, in triplicate, may be sent to any one of the editors. Please classify according to the scheme of Math. Reviews, Index to Vol. 39. Supply name and address of author to whom proofs should be sent. All other communications should be addressed to the managing editor, or Elaine Barth, University of California, Los Angeles, California, 90024.

50 reprints to each author are provided free for each article, only if page charges have been substantially paid. Additional copies may be obtained at cost in multiples of 50 .

The Pacific Journal of Mathematics is issued monthly as of January 1966. Regular subscription rate: $\$ 102.00$ a year (6 Vols., 12 issues). Special rate: $\$ 51.00$ a year to individual members of supporting institutions.

Subscriptions, orders for numbers issued in the last three calendar years, and changes of address shoud be sent to Pacific Journal of Mathematics, P.O. Box 969, Carmel Valley, CA 93924, U.S.A. Old back numbers obtainable from Kraus Periodicals Co., Route 100, Millwood, NY 10546.

\footnotetext{
PUBLISHED BY PACIFIC JOURNAL OF MATHEMATICS, A NON-PROFIT CORPORATION

Printed at Kokusai Bunken Insatsusha (International Academic Printing Co., Ltd.). 8-8, 3-chome, Takadanobaba, Shinjuku-ku, Tokyo 160, Japan.
} 


\section{Pacific Journal of Mathematics}

\section{Vol. 96, No. 2 December, 1981}

Gerald A. Beer, A natural topology for upper semicontinuous functions and

a Baire category dual for convergence in measure $\ldots \ldots \ldots \ldots \ldots \ldots 251$

Georgia Benkart and J. Marshall Osborn, An investigation of real

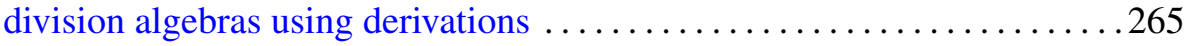

Donald Ian Cartwright and John R. McMullen, A structural criterion for the existence of infinite Sidon sets ........................ 301

Philip Hanlon, The fixed-point partition lattices $\ldots \ldots \ldots \ldots \ldots \ldots \ldots \ldots$

Eric Hayashi, The spectral density of a strongly mixing stationary Gaussian process

Chung-Wu Ho and Charles E. Morris, Jr., A graph-theoretic proof of

Sharkovsky's theorem on the periodic points of continuous functions . ...361

Sara Hurvitz, The automorphism groups of spaces and fibrations ....... 371

Atsushi Inoue, Schoichi Ota and Jun Tomiyama, Derivations of operator algebras into spaces of unbounded operators . .................. 389

Wolfgang B. Jurkat and Gary Sampson, On weak restricted estimates and endpoint problems for convolutions with oscillating kernels. I ........ 405

Georgios Koumoullis, Some topological properties of spaces of

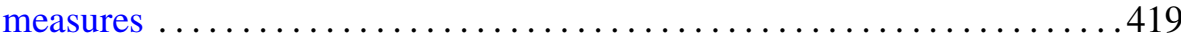

Wen Hsiung Lin, Algebraic Kahn-Priddy theorem ................. 435

Michael John McAsey, Invariant subspaces of nonselfadjoint crossed

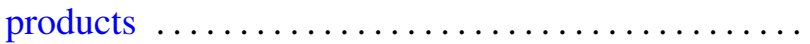

Justin Peters, Entropy of automorphisms on L.C.A. groups

Saburou Saitoh, A characterization of the adjoint $L$-kernel of Szegó type 